\title{
Withdrawal period for seeding cotton after chemical control of cotton stalks
}

\author{
Alessandra Constantin Francischini ${ }^{1}$ (1) https://orcid.org/0000-0002-5805-657 1 \\ Jamil Constantin² (D) https://orcid.org/0000-0001-6610-699X \\ Willian Daróz Matte ${ }^{2 * *}$ (D) https://orcid.org/0000-0002-4277-6910 \\ Rubem Silvério de Oliveira Junior ${ }^{2}$ (D) https://orcid.org/0000-0002-5222-8010 \\ Rafael Romero Mendes² (D) https://orcid.org/0000-0003-4404-8826 \\ Fellipe Goulart Machado² (D) https://orcid.org/0000-0003-1565-988X \\ 1. Sumitomo Chemical Latin America - São Paulo (SP) - Brazil. \\ 2. Universidade Estadual de Maringá - Departamento de Agronomia - Maringá (PR), Brazil. \\ *Corresponding author: willianmatte@hotmail.com
}

\section{ABSTRACT}

A very limited amount of information is available in relation both to the residual effect of herbicides destinated to the destruction of cotton stalks and to the time interval required to prevent that development and yield of the following crop be affected. This work aimed to identify the residual activity of herbicides intended to eliminate cotton stalks and to estimate the safety interval (SI) of time for the next cotton sowing. Two trails were simultaneously carried out, the first one for a single application and a second one for the two sequential applications of herbicide treatments, in a $15 \times 5$ factorial design organized in randomized complete blocks with four replications. Levels of first factor were constituted by herbicide treatments and the levels of the second factor were composed five periods of time for cotton sowing after herbicide application (0,30, 60, 90, and 120 days). Herbicides were 2,4-D, glyphosate, saflufenacil, [imazapic + imazapyr], dicamba, fluroxypyr and sulfentrazone. Results provided an indication of residual activity of herbicide treatments in soil and indicated that a single application or two sequential applications of glyphosate + dicamba + saflufenacil have a considerable potential to affect cotton and a period exceeding 100 days for a single application and exceeding 120 days for two sequential applications was necessary. Treatments with 2,4-D and 2,4-D + glyphosate provided the shortest safe interval and may be used for cotton stalk destruction with no risks for the crop sowing after the withdrawal period.

Keywords: carryover, Gossypium hirsutum, host-free period.

\section{INTRODUCTION}

In Brazil, herbaceous cotton (Gossypium hirsutum L. var. latifolium) is traditionally sown from November to December and harvested from June to July, mainly in the Midwest (FERREIRA et al., 2015). The crop is affected by various diseases and pest insects, which requires cotton farmers to adopt control strategies to ensure economically viable cotton production. The destruction of the postharvest cotton stalks is one of the strategies and aims to reduce the population of insect pests and disease inoculums that may remain housed in the remaining crop and cotton plants (GRIGOLLI et al., 2015; RIBEIRO et al., 2015).

The control and/or destruction of cotton crop after harvest is a recommended practice and supported by law in all Brazilian producing states, governed by federal law: Ordinances No. 75 and 77 of June 16, 1993 and No. 116 of 16 June 1994, in addition to state ordinances that implement this important prophylactic measure (ALMEIDA et al., 2008; ANDRADE JUNIOR et al., 2017). This practice is commonly performed through chemical control with herbicides, due to the agility in the process and the possibility of adopting soil conservation management (ANDRADE JUNIOR et al., 2017).

The most used herbicides in the destruction operations of cotton stalks are 2,4-D and glyphosate, applied alone or in combination, in single or sequential applications (ANDRADE JUNIOR et al., 2017). Herbicides, when applied to the

Received: July 20, 2020. Accepted: Oct 29, 2021

Associate Editor: Silvia Galleti

Peer Review History: Double-blind Peer Review. 
cotton plants, also reach the soil, and depending on the characteristics of the molecules, can be retained and remain active for a certain time (OLIVEIRA; BRIGHENTI, 2011).

The length of time some herbicide remains active in the soil and its effect on plants is called the residual activity. Some herbicides, such as glyphosate, bind so strongly to soil particles that they become unavailable to plants and have no residual activity. However, other herbicides that bind moderately to soil constituents, reach a certain balance, presenting a soil sorbed fraction and another solution of fraction and may have residual soil activity for long periods. The use of herbicides with residual activity may affect the crop sown in succession. This occurs when the crop is susceptible, the herbicide persists in the soil for longer than the interval between application and sowing of the crop and is available in soil solution in sufficient concentration to be absorbed by plants (GHENO et al., 2016; MATTE et al., 2019).

Due to the scarcity of information, this study aimed to evaluate the residual activity promoted by herbicides used in the management of the destruction of cotton stalks and to estimate the safety interval (SI) for sowing cotton in succession.

\section{MATERIAL AND METHODS}

The experiments were conducted in a greenhouse in the municipality of Santo Antônio de Posse, state of São Paulo $\left(22^{\circ} 36^{\prime} 13.5^{\prime \prime} \mathrm{S}\right.$ and $46^{\circ} 59^{\prime} 05.7^{\prime \prime} \mathrm{W}$, altitude of $\left.658 \mathrm{~m}\right)$ from April to December 2014. The average temperatures observed during the conduction period of the experiments are presented in Figure 1.

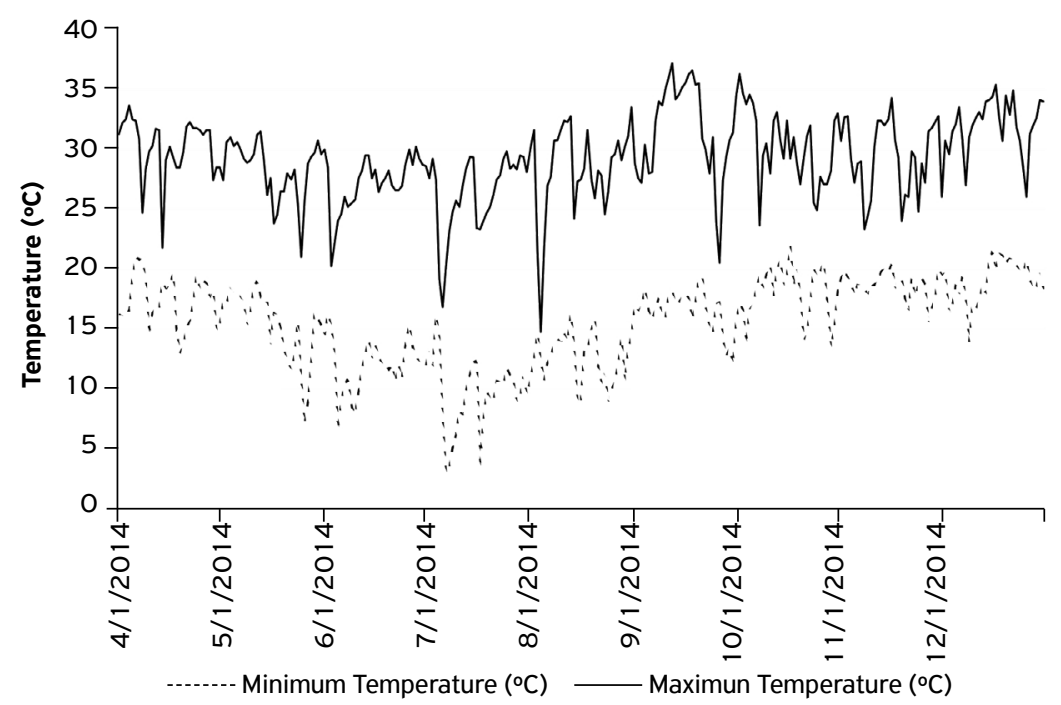

Figure 1. Minimum and maximum temperature $\left({ }^{\circ} \mathrm{C}\right)$ observed during the conduction period of the experiments. Santo Antônio de Posse, SP, 2014.

Source: Elaborated by the authors.

Two experiments were conducted simultaneously, one for a single application (experiment 1 ) and one for sequential application (experiment 2 ) of herbicide treatments. The experiments were installed in a $15 \times 5$ factorial scheme, in outlining of randomized blocks with four replications. The first factor evaluated was herbicide treatments (Table 1 ) and the second factor was five sowing times of FiberMax 966 cotton (0, 30, 60, 90, and 120 days) after application A in experiment 1, and after application B in experiment 2.

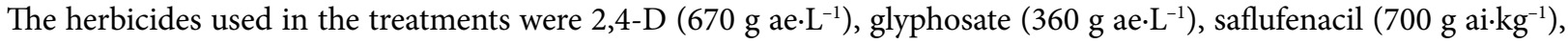
[imazapic + imazapyr] $\left(\left[175 \mathrm{~g}+525 \mathrm{~g}\right.\right.$ ae $\left.\left.\cdot \mathrm{kg}^{-1}\right]\right)$, dicamba $\left(480 \mathrm{~g}\right.$ ae $\left.\cdot \mathrm{L}^{-1}\right)$, fluroxypyr $\left(200 \mathrm{~g}\right.$ ae $\left.\cdot \mathrm{L}^{-1}\right)$ and sulfentrazone $\left(500 \mathrm{~g}\right.$ ai $\left.\cdot \mathrm{L}^{-1}\right)$.

The experimental plots were composed of $3 \mathrm{dm}^{3}$ plastic pots filled with dry and sieved soil. According to chemical and physical analysis, the soil used had the following characteristics: $\mathrm{pH}$ in $\mathrm{CaCl}_{2}$ of $5.4 ; 18.7 \mathrm{mmol}_{c} \cdot \mathrm{dm}^{-3} \mathrm{of} \mathrm{H}^{+}+\mathrm{Al}^{+3}$; $40.3 \mathrm{mg} \cdot \mathrm{dm}^{-3}$ of $\mathrm{P} ; 50.0 \mathrm{mmol}_{c} \cdot \mathrm{dm}^{-3}$ of $\mathrm{Ca}^{+2} ; 28.7 \mathrm{mmol}_{c} \cdot \mathrm{dm}^{-3}$ of $\mathrm{Mg}^{+2} ; 2.4 \mathrm{mmol}_{c} \cdot \mathrm{dm}^{-3}$ of $\mathrm{K}^{+}$; cation exchange capacity at $\mathrm{pH} 7.0$ of $99.8 \mathrm{mmol} \cdot \mathrm{dm}^{-3} ; 27 \mathrm{~g} \cdot \mathrm{dm}^{-3}$ of organic matter; $15 \%$ coarse sand; $33 \%$ fine sand; $5 \%$ silt; and $47 \%$ clay.

In the first experiment, the single application of herbicide treatments (A) was performed on May 27, 2014. On the same date, cotton was sown for zero days after application (0 DAA). Subsequent sowing was performed on May 28, 2014 (30 DAA), June 27, 2014 (60 DAA), July 27, 2014 (90 DAA) and August 27, 2014 (120 DAA). Soon after each application 
of herbicide treatments, all pots received irrigation with a 5-mm water depth. After irrigation, the pots that were not sown remained without irrigation until they received cotton sowing and the pots sown started to receive irrigation with a daily 5-mm water depth until the end of the experiment.

Table 1. List of herbicide treatments used to evaluate the "carryover" effect in cotton culture after one (application A) and two sequential applications (application A/B) of the treatments. Santo Antônio de Posse, SP, 2014.

\begin{tabular}{lc}
\hline Herbicide treatment & $\begin{array}{c}\text { Application A and B } \\
\text { Dose ai or, and ae g·ha }{ }^{-1}\end{array}$ \\
\hline $2,4-D$ & 1340 \\
\hline $2,4-D+$ glyphosate & $1340+720$ \\
\hline 2,4-D + saflufenacil & $1340+105$ \\
\hline 2,4-D + [imazapic + imazapyr] & $1340+105$ \\
\hline 2,4-D + glyphosate + saflufenacil & $1340+720+105$ \\
\hline 2,4-D + glyphosate + [imazapic + imazapyr] & $1340+720+105$ \\
\hline 2,4-D + glyphosate + saflufenacil + [imazapic + imazapyr] & $1340+720+105+105$ \\
\hline Glyphosate + saflufenacil + [imazapic + imazapyr] & $720+105+105$ \\
\hline Glyphosate + saflufenacil + Fluroxypyr & $720+105+400$ \\
\hline Glyphosate + fluroxypyr + [imazapic + imazapyr] & $720+400+105$ \\
\hline Glyphosate + dicamba + saflufenacil & $720+960+105$ \\
\hline Glyphosate + sulfentrazone + 2,4-D & $720+40+1340$ \\
\hline Sulfentrazone + 2,4-D & $40+1340$ \\
\hline Fluroxypyr & 400 \\
\hline Control without application & - \\
\hline All & 420
\end{tabular}

All herbicidal treatments were applied in association with Dash $\mathrm{HC} 0.5 \% \mathrm{v} \cdot \mathrm{v}^{-1}$; ai: active ingredient; ae: acid equivalent.

Source: Elaborated by the authors.

In the second experiment, two sequential applications of the same herbicide treatments (A/B applications) were performed. The first application (A) was performed on April 27, 2014 and the second application (B) was performed 45 days after the first (June 11,2014). After application A of the herbicide treatments, the pots were irrigated with a 5-mm water depth and were without irrigation for 45 days until the second application (B). After the second application (B), a new irrigation with 5 -mm depth was performed. The pots that did not receive cotton sowing remained without irrigation until they were sown. From sowing, the pots received a daily 5-mm water depth until the end of the experiment.

The 45-day interval between the first and the second application was stipulated simulating the time required for the cotton plants to sprout in the field and to present sufficient leaf area for the absorption of herbicide treatments in the second application (FRANCISCHINI et al., 2019). The absence of irrigation in the pots after the application aimed to simulate a common situation in cotton producing areas, in which there are pronounced periods of drought after harvest, during the period destined to the control of the cotton stalks. Thus, for the experiment with two sequential applications (A/B), the cotton sowing was performed on June 11, 2014 (0 DAA), July 4, 2014 (30 DAA), August 4, 2014 (60 DAA), September 4, 2014 (90 DAA) and October 4, 2014 (120 DAA).

For all applications, a $\mathrm{CO}_{2}$-based constant pressure costal sprayer equipped with three XR-110.02 fan blades, spaced $50 \mathrm{~cm}$ apart under pressure of $2.0 \mathrm{kgf} \cdot \mathrm{cm}^{-2}$, was used. These application conditions provided the equivalent of $150 \mathrm{~L} \cdot \mathrm{ha} \mathrm{a}^{-1}$ of syrup. The climatic conditions at the time of application of the treatments were: (A) relative humidity $67 \%$, temperature $25.9^{\circ} \mathrm{C}$, wind speed $0.8 \mathrm{~km} \cdot \mathrm{h}^{-1}$ and moist soil; (B) relative humidity $79 \%$, temperature $26.8^{\circ} \mathrm{C}$, wind speed $0.6 \mathrm{~km} \cdot \mathrm{h}^{-1}$ and moist soil.

Cotton poisoning was evaluated 14 days after sowing (DAS) using a percentage scale from zero to one hundred, where $0 \%$ represents no symptoms and $100 \%$ represents plant death. At the end of the experiment, at 30 DAS, plant height and dry weight of the aerial part of cotton were evaluated. The dry mass and height data were corrected to percentage values compared to the control without application, assuming that the pots that remained without herbicide application were $100 \%$ of the height and produced $100 \%$ of dry mass.

The data were initially subjected to analysis of variance and, when F values were significant (p-value $\leq 0.05$ ), regression models were adjusted for each variable analyzed, presented in Table 2. 
Table 2. Regression models adjusted to estimate the safety interval for cotton sowing. Santo Antônio de Posse, SP, 2014.

\section{Regression models}

Boltzmann's sigmoidal model (3 parameters)

$$
y=\frac{a}{1+e^{-\left(x-\frac{x 0}{b}\right)}}
$$

Boltzmann's sigmoidal model (4 parameters)

$$
y=y 0+\frac{a-y 0}{1+e^{-\left(x-\frac{x 0}{b}\right)}}
$$

Streibig logistic model (3 parameters)

$$
y=\frac{a}{\left[1+\left(\frac{x}{x 0}\right)^{b}\right]}
$$

Streibig logistic model (4 parameters)

$$
y=y 0+\frac{a}{\left[1+\left(\frac{x}{x 0}\right)^{b}\right]}
$$

Exponential model (2 parameters)

$$
y=a e^{-b x}
$$

Exponential model (3 parameters)

$$
y=a|x-x 0|^{b}
$$

$y=$ height, biomass or intoxication; $x=$ days after sowing; $a, x O$ and $b=$ estimated parameter of the equation such that: $y O=$ minimum height, biomass or intoxication value; $a=$ maximum asymptote of function (maximum height, biomass or intoxication); $x O=$ "days" providing $50 \%$ of the value of " $a$ "; $b$ = slope of the curve around $x O$.

Source: Elaborated by the authors.

Based on the data obtained, it was possible to determine the number of days required after the single and sequential application for the cotton sowing, without risk of interference of herbicide treatments used in the destruction of the cotton stalks in the previous cycle. This period of days was called the safety interval (SI).

To determine the SI for cotton sowing after herbicide treatments, the following criteria were considered tolerable: phytointoxication - acceptable up to $10 \%$; plant height - maximum reduction of $10 \%$ in relation to the control height; relative dry mass - maximum reduction of $10 \%$ in relation to the control dry mass. In the end, the highest value obtained among the three analyzed variables was chosen as SI.

\section{RESULTS AND DISCUSSION}

There was a significant interaction of herbicide treatments and cotton sowing season for all variables analyzed when a single herbicide application was performed. The curves shown in Figure $2 \mathrm{a}-\mathrm{c}$ were adjusted according to the regression models presented in Table 2, which equations are shown in Table 3.

All herbicide treatments when applied on sowing day (zero days) promoted cotton poisoning (Fig. 2a). The lowest levels of phytointoxication were observed with the application of 2,4-D, being the only treatment that promoted less than $10.0 \%$ intoxication. In the sowing performed at $30 \mathrm{DAA}$, in addition to 2,4-D, treatments with 2,4-D + glyphosate and fluroxypyr also started presenting cotton intoxication of less than $10.0 \%$.

For the cotton sown at $60 \mathrm{DAA}$, the herbicide treatments that promoted some intoxication but less than $10.0 \%$ were: 2,4-D + glyphosate, 2,4-D + saflufenacil, 2,4-D + glyphosate + saflufenacil. and glyphosate + [imazapic + imazapyr] + fluroxypyr. At sowing performed at 90 DAA, the treatments 2,4-D + [imazapic + imazapyr] and glyphosate + dicamba + saflufenacil promoted more than $10.0 \%$ of cotton intoxication. When cotton was sown at 120 DAA, some treatments still promoted some intoxication, but the values were lower than those stipulated as tolerable in this study $(<10.0 \%)$.

In general, most treatments affected cotton height when sowing was performed up to 60 days (Fig. $2 \mathrm{~b}$ ). The only treatment that did not affect cotton growth under any conditions was 2,4-D. For sowing at 30 days, in addition to this 
treatment, treatments with 2,4-D + glyphosate and fluroxypyr did not significantly affect cotton height. The other herbicide treatments provided negative effects on cotton height for longer period (60 days). In sowing performed at 120 days, no herbicide treatment affected the height of cotton plants.
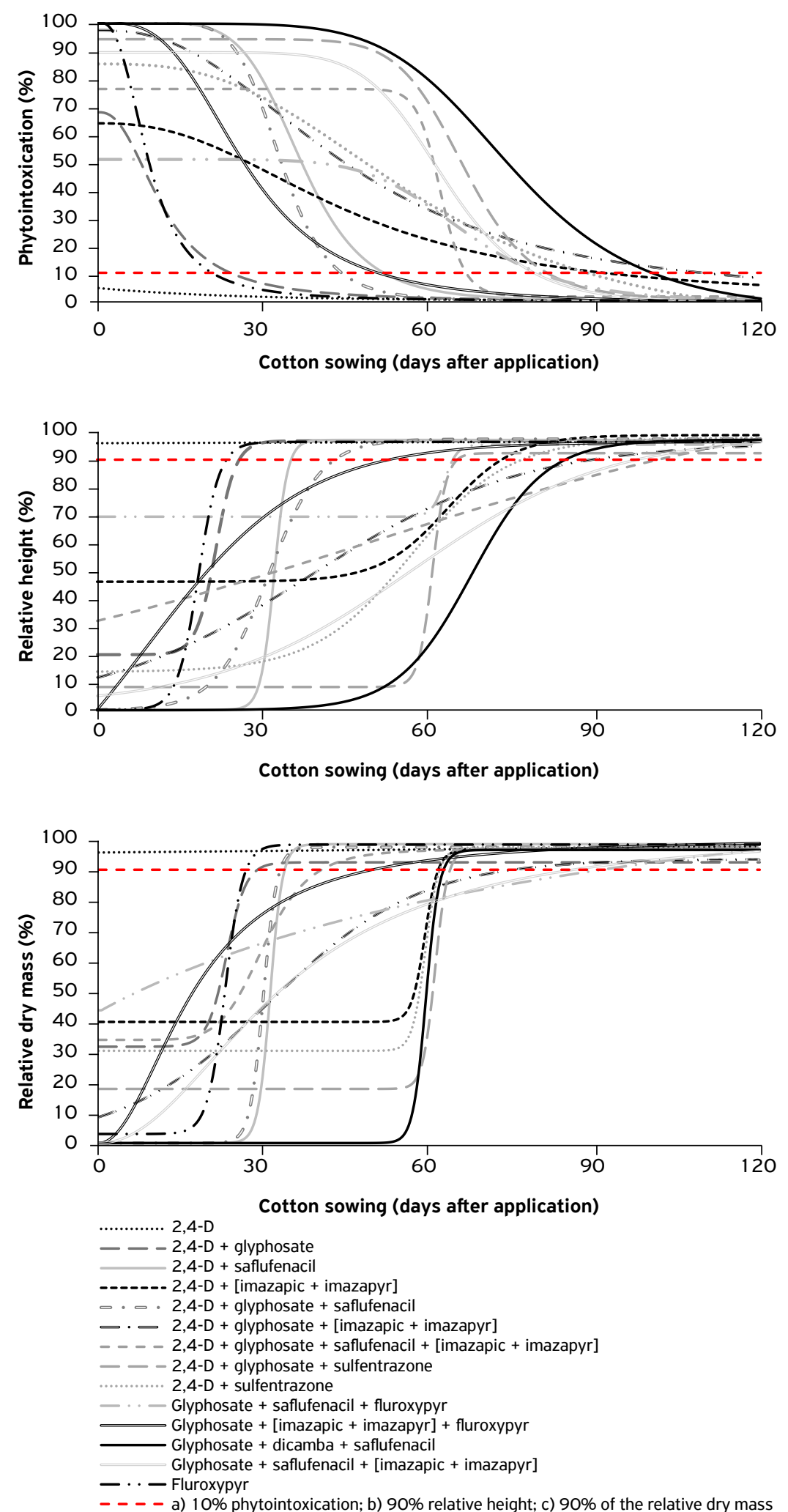

Figure 2. Phytointoxication (a), relative height (b) and relative dry mass (c) of cotton planted at $0,30,60,90$, and 120 days after single application (application A) of herbicide treatments used in the control of cotton stalks. Santo Antônio de Posse, SP, 2014. Source: Elaborated by the authors. 
Table 3. Equations used to estimate the safety interval aiming at cotton sowing after herbicide application in the control of cotton stalks. Santo Antônio de Posse, SP, 2014.

\begin{tabular}{|c|c|c|c|}
\hline \multirow{3}{*}{ Treatments (application A) } & \multicolumn{3}{|c|}{ Equations used to estimate the safety interval for cotton sowing } \\
\hline & \multicolumn{3}{|c|}{ Phytointoxication } \\
\hline & & Relative height & Relative dry mass \\
\hline 2,4-D & $\hat{Y}=4.51^{*} \mathrm{EXP}\left(-0.04^{*} \mathrm{x}\right)$ & - & - \\
\hline 2,4-D + glyphosate & $\begin{array}{c}\hat{Y}=-0.34+(68.09 / \\
\left.\left(1+\left((x / 11.66)^{\wedge} 2.4\right)\right)\right)\end{array}$ & - & - \\
\hline 2,4-D + saflufenacil & $\begin{array}{c}\hat{Y}=-0.18+(100.17 / \\
\left.\left(1+\left((x / 36.74)^{\wedge} 6.39\right)\right)\right)\end{array}$ & $\begin{array}{l}\hat{Y}=19.82+76.96 /(1+ \\
\operatorname{EXP}(-(x-21.51) / 1.79))\end{array}$ & $\begin{array}{l}\hat{Y}=31.73+60.71 /(1+ \\
\operatorname{EXP}(-(x-23.24) / 1.76))\end{array}$ \\
\hline $\begin{array}{l}\text { 2,4-D + [imazapic + } \\
\text { imazapyr] }\end{array}$ & $\begin{array}{c}\hat{Y}=63.76 / \\
\left.\left(1+\left((x / 45.65)^{\wedge} 2.42\right)\right)\right)\end{array}$ & $\begin{array}{c}\hat{Y}=\left(-3.4^{*} 10^{\wedge}-10\right)+97.09 / \\
(1+\operatorname{EXP}(-(x-32.01) / 1.21))\end{array}$ & $\begin{array}{c}\hat{Y}=\left(-6.7^{*} 10^{\wedge}-11\right)+98.42 / \\
(1+\operatorname{EXP}(-(x-31.14) / 1.11))\end{array}$ \\
\hline $\begin{array}{l}2,4-D+\text { glyphosate }+ \\
\text { saflufenacil }\end{array}$ & $\begin{array}{c}\hat{Y}=-0.025+(100.02 / \\
\left.\left(1+\left((x / 33.05)^{\wedge} 7.66\right)\right)\right)\end{array}$ & $\begin{array}{l}\hat{Y}=46.07+52.77 /(1+ \\
\operatorname{EXP}(-(x-63.39) / 6.09))\end{array}$ & $\begin{array}{l}\hat{Y}=39.89+56.66 /(1+ \\
\operatorname{EXP}(-(x-59.21) / 1.2))\end{array}$ \\
\hline $\begin{array}{l}\text { 2,4-D + glyphosate } \\
+[\text { imazapic + imazapyr }]\end{array}$ & $\begin{array}{c}\hat{Y}=97.21 / \\
\left.\left(1+\left((x / 45.35)^{\wedge} 2.46\right)\right)\right)\end{array}$ & $\begin{array}{l}\hat{Y}=-0.15+97.58 /(1+ \\
\operatorname{EXP}(-(x-31.02) / 4.79))\end{array}$ & $\begin{array}{l}\hat{Y}=\left(-2.98^{*} 10^{\wedge}-9\right)+97.44 / \\
(1+\operatorname{EXP}(-(x-29.91) / 1.23))\end{array}$ \\
\hline $\begin{array}{l}\text { 2,4-D + glyphosate + saflufenacil } \\
+ \text { [imazapic + imazapyr }]\end{array}$ & $\begin{array}{c}\hat{Y}=1.49+(74.62 / \\
\left.\left(1+\left((x / 62)^{\wedge} 28.71\right)\right)\right)\end{array}$ & $\begin{array}{c}\hat{Y}=96.36 /(1+ \\
\operatorname{EXP}(-(x-38.44) / 19.26))\end{array}$ & $\begin{array}{c}\hat{Y}=93.53 /(1+ \\
\operatorname{EXP}(-(x-31.2) / 13.59))\end{array}$ \\
\hline $\begin{array}{l}2,4-D+\text { glyphosate }+ \\
\text { sulfentrazone }\end{array}$ & $\begin{array}{c}\hat{Y}=0.32+(93.68 / \\
\left.\left(1+\left((x / 66.42)^{\wedge} 10.74\right)\right)\right)\end{array}$ & $\begin{array}{c}\hat{Y}=2.66=115.52 /(1+ \\
\operatorname{EXP}(-(x-49.50) / 45.92))\end{array}$ & $\begin{array}{c}\hat{Y}=33.98+(62.76 / \\
\left.\left(1+\left((x / 30.09)^{\wedge}-7.16\right)\right)\right)\end{array}$ \\
\hline 2,4-D + sulfentrazone & $\begin{array}{c}\hat{Y}=-13.81+(98.92 / \\
\left.\left(1+\left((x / 59.23)^{\wedge} 2.86\right)\right)\right)\end{array}$ & $\begin{array}{c}\hat{Y}=8.19+84.14 /(1+ \\
\operatorname{EXP}(-(x-60.65) / 1.26))\end{array}$ & $\begin{array}{c}\hat{Y}=17.82+(79.11 / \\
\left.\left(1+\left((x / 61.08)^{\wedge}-53.95\right)\right)\right)\end{array}$ \\
\hline $\begin{array}{l}\text { Glyphosate + saflufenacil + } \\
\text { fluroxypyr }\end{array}$ & $\begin{array}{c}\hat{Y}=-0.52+(51.30 / \\
\left.\left(1+\left((x / 66.1)^{\wedge} 7.23\right)\right)\right)\end{array}$ & $\begin{array}{l}\hat{Y}=13.58+84.29 /(1+ \\
\operatorname{EXp}(-(x-56.81) / 8.81))\end{array}$ & $\begin{array}{c}\hat{Y}=30.38+(67.05 / \\
\left.\left(1+\left((x / 59.35)^{\wedge}-49.04\right)\right)\right)\end{array}$ \\
\hline $\begin{array}{l}\text { Glyphosate + [imazapic + } \\
\text { imazapyr] + fluroxypyr }\end{array}$ & $\begin{array}{c}\hat{Y}=-1.27+(101.27 / \\
\left.\left(1+\left((x / 26.44)^{\wedge} 3.2\right)\right)\right)\end{array}$ & $\begin{array}{l}\hat{Y}=69.48+25.92 /(1+ \\
\operatorname{EXP}(-(x-62.82) / 1.14))\end{array}$ & $\begin{array}{c}\hat{Y}=43.23+(98.73 / \\
\left.\left(1+\left((x / 101.5)^{\wedge}-0.99\right)\right)\right)\end{array}$ \\
\hline $\begin{array}{l}\text { Glyphosate + dicamba + } \\
\text { saflufenacil }\end{array}$ & $\begin{array}{c}\hat{Y}=-4.38+(104 / \\
\left.\left(1+\left((x / 74.96)^{\wedge} 6.29\right)\right)\right)\end{array}$ & $\begin{array}{c}\hat{Y}=-51.73+148.18 / \\
(1+\operatorname{EXP}(-(x-8.85) / 14.22))\end{array}$ & $\begin{array}{c}\hat{Y}=100.72 /(1+ \\
\left.\left((x / 17.32)^{\wedge}-2.02\right)\right)\end{array}$ \\
\hline $\begin{array}{l}\text { Glyphosate + saflufenacil + } \\
\text { [imazapic + imazapyr] }\end{array}$ & $\begin{array}{c}\hat{Y}=-1.27+(90.55 / \\
\left.\left(1+\left((x / 62.59)^{\wedge} 8.43\right)\right)\right)\end{array}$ & $\begin{array}{c}\hat{Y}=-0.17+97.34 /(1+ \\
\operatorname{EXP}(-(x-67.70) / 6.70))\end{array}$ & $\begin{array}{c}\hat{Y}=\left(1.13^{*} 10^{\wedge}-6\right)+(96.53 / \\
\left.\left(1+\left((x / 59.44)^{\wedge}-50.31\right)\right)\right)\end{array}$ \\
\hline Fluroxypyr & $\begin{array}{c}\hat{Y}=-0.14+(100.14 / \\
\left.\left(1+\left((x / 9.12)^{\wedge} 2.69\right)\right)\right)\end{array}$ & $\begin{array}{c}\hat{Y}=1.14+97.87 /(1+ \\
\operatorname{EXP}(-9 x-56.86) / 17.80))\end{array}$ & $\begin{array}{c}\hat{Y}=103.08 /(1+ \\
\left.\left((x / 33.77)^{\wedge}-2.06\right)\right)\end{array}$ \\
\hline Control without application & - & $\begin{array}{c}\hat{Y}=-0.07+96.46 /(1+ \\
\operatorname{EXP}(-(x-18.31) / 1.92))\end{array}$ & $\begin{array}{c}\hat{Y}=2.99+(95.3 /(1+ \\
\operatorname{EXP}(-(x-22.97) / 1.62)))\end{array}$ \\
\hline \multicolumn{4}{|l|}{ Treatments (application A/B) } \\
\hline $2,4-D$ & $\begin{array}{c}\hat{Y}=-2.05+39.80 / \\
\left(1+\left((x / 2.47)^{\wedge} 0.77\right)\right)\end{array}$ & $\begin{array}{c}\hat{Y}=96.675 /(1+ \\
\operatorname{EXP}(-(x+1.84) / 1.4758))\end{array}$ & $\begin{array}{c}\hat{Y}=97.36 /(1+ \\
\operatorname{EXP}(-(x+1.78) / 1.46))\end{array}$ \\
\hline 2,4-D + glyphosate & $\begin{array}{c}\hat{Y}=-0.73+32.73 / \\
\left(1+\left((x / 15.447)^{\wedge} 1.96\right)\right)\end{array}$ & $\begin{array}{c}\hat{Y}=96.925 /(1+ \\
\operatorname{EXP}(-(x+2.00) / 1.459))\end{array}$ & $\begin{array}{c}\hat{Y}=98.5 /(1+ \\
\operatorname{EXP}(-(x+1.66) / 1.34))\end{array}$ \\
\hline 2,4-D + saflufenacil & $\begin{array}{c}\hat{Y}=-2.4+72.14 / \\
\left(1+\left((x / 30.38)^{\wedge} 2.73\right)\right)\end{array}$ & $\begin{array}{c}\hat{Y}=38.74+58.13 / \\
\left(1+\left((x / 28.38)^{\wedge}-6.09\right)\right)\end{array}$ & $\begin{array}{c}\hat{Y}=39.86+57.45 / \\
\left.\left(1+\left((x / 29.21)^{\wedge}-28.77\right)\right)\right)\end{array}$ \\
\hline $\begin{array}{l}\text { 2,4-D + [imazapic + } \\
\text { imazapyr] }\end{array}$ & $\begin{array}{c}\hat{Y}=-5.42+105.34 / \\
\left(1+\left((x / 45.02)^{\wedge} 3.14\right)\right)\end{array}$ & $\begin{array}{c}\hat{Y}=96.84 /(1+ \\
\left.\left((x / 37.17)^{\wedge}-4.05\right)\right)\end{array}$ & $\begin{array}{c}\hat{Y}=103.24 /(1+ \\
\left.\left((x / 37.03)^{\wedge}-2.78\right)\right)\end{array}$ \\
\hline $\begin{array}{l}\text { 2,4-D + glyphosate + } \\
\text { saflufenacil }\end{array}$ & $\begin{array}{c}\hat{Y}=-0.38+76.88 / \\
\left(1+\left((x / 27.26)^{\wedge} 4.02\right)\right)\end{array}$ & $\begin{array}{c}\hat{Y}=29.73+68.75 / \\
\left(1+\left((x / 15.61)^{\wedge}-1.76\right)\right)\end{array}$ & $\begin{array}{c}\hat{Y}=33+73.48 / \\
\left(1+\left((x / 25.65)^{\wedge}-1.44\right)\right)\end{array}$ \\
\hline $\begin{array}{l}\text { 2,4-D + glyphosate } \\
+[\text { imazapic + imazapyr }]\end{array}$ & $\begin{array}{c}\hat{Y}=99.66 / \\
\left(1+\left((x / 30.50)^{\wedge} 2.21\right)\right)\end{array}$ & $\begin{array}{c}\hat{Y}=100.99 /(1+ \\
\left.\left((x / 26.03)^{\wedge}-1.88\right)\right)\end{array}$ & $\begin{array}{c}\hat{Y}=145.17 /(1+ \\
\left.\left((x / 41.84)^{\wedge}-0.72\right)\right)\end{array}$ \\
\hline $\begin{array}{l}\text { 2,4-D + glyphosate + } \\
\text { saflufenacil + [imazapic + } \\
\text { imazapyr] }\end{array}$ & $\begin{array}{c}\hat{Y}=99.06 / \\
\left(1+\left((x / 31.65)^{\wedge} 2.34\right)\right)\end{array}$ & $\begin{array}{c}\hat{Y}=104.19 /(1+ \\
\left.\left((x / 27.98)^{\wedge}-1.85\right)\right)\end{array}$ & $\begin{array}{c}\hat{Y}=137.33 /(1+ \\
\left.\left((x / 40.92)^{\wedge}-0.94\right)\right)\end{array}$ \\
\hline $\begin{array}{l}2,4-D+\text { glyphosate }+ \\
\text { sulfentrazone }\end{array}$ & $\begin{array}{c}\hat{Y}=-8.26+107.89 / \\
\left(1+\left((x / 48.04)^{\wedge} 3.13\right)\right)\end{array}$ & $\begin{array}{c}\hat{Y}=101.6 /(1+ \\
\left.\left((x / 41.17)^{\wedge}-3.19\right)\right)\end{array}$ & $\begin{array}{c}\hat{Y}=111.25 /(1+ \\
\left.\left((x / 44.60)^{\wedge}-2.31\right)\right)\end{array}$ \\
\hline
\end{tabular}


Table 3. Continuation...

\begin{tabular}{|c|c|c|c|}
\hline \multirow{3}{*}{$\begin{array}{l}\text { Treatments } \\
\text { (application A/B) }\end{array}$} & \multicolumn{3}{|c|}{ Equations used to estimate the safety interval for cotton sowing } \\
\hline & \multicolumn{3}{|c|}{ Phytointoxication } \\
\hline & & Relative height & Relative dry mass \\
\hline 2,4-D + sulfentrazone & $\begin{array}{c}\hat{Y}=92.91 / \\
\left(1+\left((x / 37.40)^{\wedge} 2.36\right)\right)\end{array}$ & $\begin{array}{c}\hat{Y}=8.92+93.38 / \\
\left(1+\left((x / 31.82)^{\wedge}-2.42\right)\right)\end{array}$ & $\begin{array}{c}\hat{Y}=9.69+94.61 / \\
\left(1+\left((x / 30.17)^{\wedge}-2.26\right)\right)\end{array}$ \\
\hline $\begin{array}{l}\text { Glyphosate + saflufenacil + } \\
\text { fluroxypyr }\end{array}$ & $\begin{array}{c}\hat{Y}=-11.22+185.05 / \\
(1+\operatorname{EXP}(-(x-9.82) /-37.79))\end{array}$ & $\begin{array}{c}\hat{Y}=103.47 /(1+ \\
\left.\left((x / 31.98)^{\wedge}-2.39\right)\right)\end{array}$ & $\begin{array}{c}\hat{Y}=36.87+75.32 / \\
\left(1+\left((x / 46.05)^{\wedge}-1.65\right)\right)\end{array}$ \\
\hline $\begin{array}{l}\text { Glyphosate + [imazapic + } \\
\text { imazapyr] + fluroxypyr }\end{array}$ & $\begin{array}{c}\hat{Y}=-10.13+109.97 / \\
\left(1+\left((x / 42.30)^{\wedge} 2.46\right)\right)\end{array}$ & $\begin{array}{c}\hat{Y}=33.24+68.33 / \\
\left(1+\left((x / 37.85)^{\wedge}-2.12\right)\right)\end{array}$ & $\begin{array}{c}\hat{Y}=115.59 /(1+ \\
\left.\left((x / 33.14)^{\wedge}-1.46\right)\right)\end{array}$ \\
\hline $\begin{array}{l}\text { Glyphosate + dicamba + } \\
\text { saflufenacil }\end{array}$ & $\begin{array}{c}\hat{Y}=19.04+81.46 / \\
\left(1+\left((x / 85.89)^{\wedge} 4.05\right)\right)\end{array}$ & $\begin{array}{c}\hat{Y}=-1.99+95.7 / \\
\left(1+\left((x / 78.23)^{\wedge}-2.95\right)\right)\end{array}$ & $\begin{array}{c}\hat{Y}=-1.99+140.14 / \\
\left(1+\left((x / 110)^{\wedge}-2.38\right)\right)\end{array}$ \\
\hline $\begin{array}{l}\text { Glyphosate + saflufenacil + } \\
\text { [imazapic + imazapyr] }\end{array}$ & $\begin{array}{c}\hat{Y}=-0.44+100.44 / \\
\left(1+\left((x / 25.73)^{\wedge} 3.63\right)\right)\end{array}$ & $\begin{array}{c}\hat{Y}=97.02 /(1+ \\
\left.\left((x / 28.94)^{\wedge}-6.14\right)\right)\end{array}$ & $\begin{array}{c}\hat{Y}=97.46 /(1+ \\
\left.\left((x / 26.03)^{\wedge}-3.57\right)\right)\end{array}$ \\
\hline Fluroxypyr & $\begin{array}{c}\hat{Y}=0.13+80.37 /(1+ \\
\operatorname{EXP}(-(x-25.14) /-10.88))\end{array}$ & $\begin{array}{c}\hat{Y}=26.11+70.29 / \\
\left(1+\left((x / 29.46)^{\wedge}-26.63\right)\right)\end{array}$ & $\begin{array}{c}\hat{Y}=29.99+70.59 / \\
\left(1+\left((x / 26.76)^{\wedge}-2.61\right)\right)\end{array}$ \\
\hline
\end{tabular}

Source: elaborated by the authors.

The application of herbicide treatments reduced dry weight of cotton plants, especially when the interval between application and sowing was less than or equal to 60 days for most herbicide treatments (Fig. 2c). The only treatment that did not promote reduction of dry mass at sowing performed at zero days was 2,4-D. For sowing at 30 days, besides the 2,4-D treatment, the 2,4-D + glyphosate and fluroxypyr applications also no longer affected the dry weight of cotton. In the sowing performed at 90 and 120 days no effect of herbicide treatments on the dry mass of cotton was observed, and the cotton plants, regardless of the treatment, presented at least $90.0 \%$ of dry mass in relation to the control.

Based on the obtained data, the SI for cotton sowing was estimated after the application A of herbicide treatments. Considering the maximum tolerances proposed in this study (10\% reduction compared to the control without herbicide) and the adjusted curves, the SI values for the single application of the treatments were calculated (Table 4). The lowest SI for cotton sowing were observed in treatments with 2,4-D (0 days), fluroxypyr (26 days) and 2,4-D + glyphosate (29 days). On the other hand, the treatments with higher residual activity were 2,4-D + glyphosate + [imazapic + imazapyr] (102 days), 2,4-D + glyphosate + saflufenacil + [imazapic + imazapyr] (101 days) and glyphosate + dicamba + saflufenacil (100 days).

Table 4. Estimated safety interval after a single application of herbicide treatments (A) for cotton sowing based on phytointoxication, height and dry mass data. Experiment 1. Santo Antônio de Posse, SP, 2014.

\begin{tabular}{|c|c|c|c|}
\hline \multirow{2}{*}{ Treatments (application A) } & \multicolumn{3}{|c|}{$\begin{array}{l}\text { Safety interval (SI) for the cotton sowing } \\
\text { (days after application) }{ }^{1}\end{array}$} \\
\hline & Phytointoxication & Height & Dry mass \\
\hline 2,4-D & 0 & 0 & 0 \\
\hline 2,4-D + glyphosate & 23 & 25 & 29 \\
\hline 2,4-D + saflufenacil & 51 & 35 & 33 \\
\hline 2,4-D + [imazapic + imazapyr] & 90 & 73 & 61 \\
\hline 2,4-D + glyphosate + saflufenacil & 44 & 43 & 32 \\
\hline 2,4-D + glyphosate + [imazapic + imazapyr] & 102 & 89 & 75 \\
\hline 2,4-D + glyphosate + saflufenacil + [imazapic + imazapyr $]$ & 66 & 101 & 40 \\
\hline 2,4-D + glyphosate + sulfentrazone & 81 & 65 & 63 \\
\hline 2,4-D + sulfentrazone & 89 & 77 & 62 \\
\hline Glyphosate + saflufenacil + fluroxypyr & 79 & 64 & 91 \\
\hline Glyphosate + [imazapic + imazapyr] + fluroxypyr & 50 & 52 & 49 \\
\hline Glyphosate + dicamba + saflufenacil & 100 & 84 & 62 \\
\hline Glyphosate + saflufenacil + [imazapic + imazapyr] & 78 & 97 & 85 \\
\hline Fluroxypyr & 20 & 23 & 26 \\
\hline Control without application & - & - & - \\
\hline
\end{tabular}


There was a significant interaction of herbicide treatments and cotton sowing season when two sequential applications (A/B) of the treatments were performed. The curves shown in Figure 3a-c were adjusted according to the regression models presented in Table 2, whose equations are shown in Table 3.
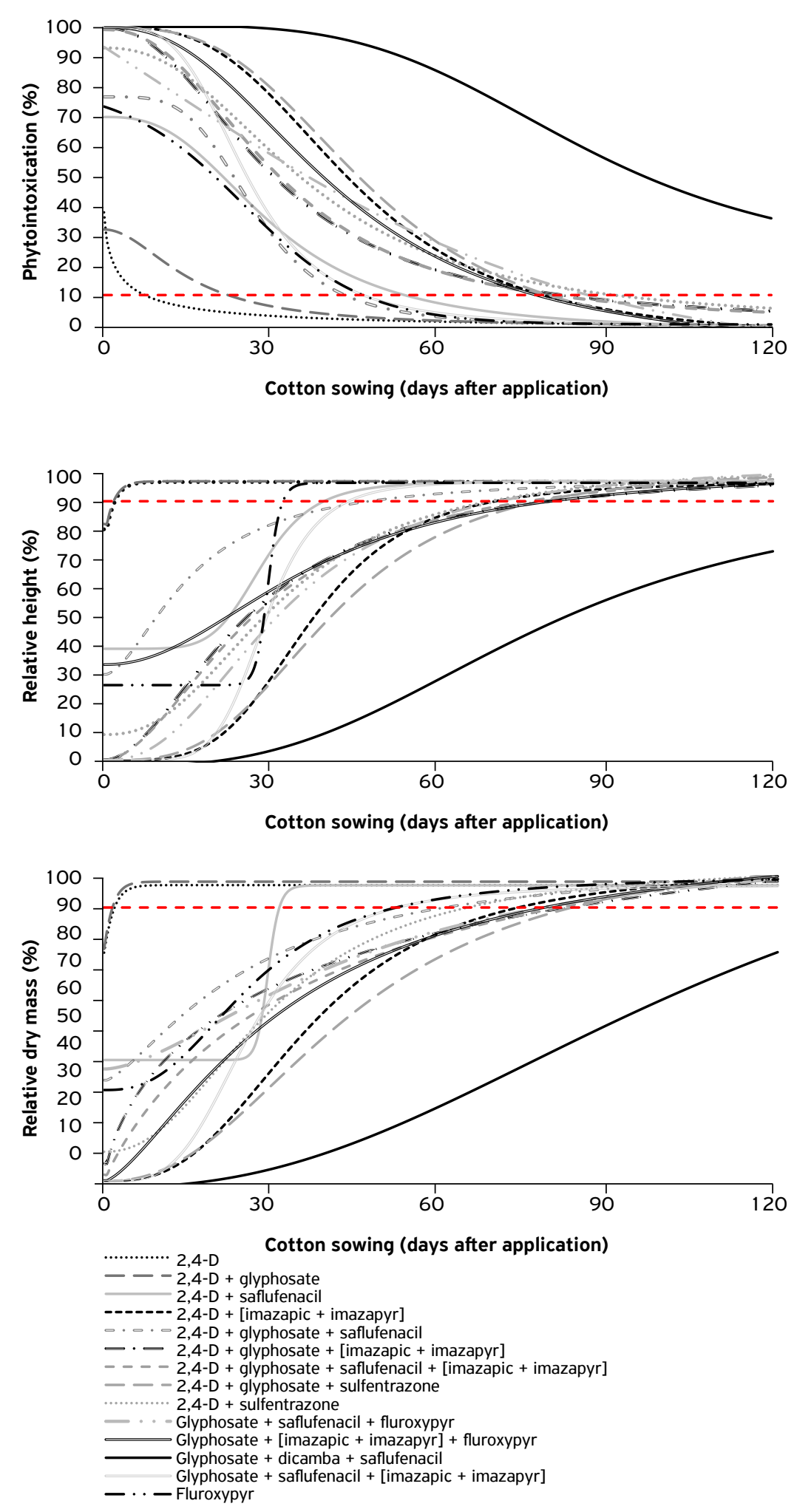

Figure 3. Phytointoxication (a), relative height (b) and relative dry weight (c) of cotton planted at $0,30,60,90$, and 120 days after sequential application (A/B application) of herbicide control treatments for cotton stalks. Santo Antônio de Posse, SP, 2014. Source: Elaborated by the authors. 
When two sequential applications of herbicide treatments (A/B) were performed, higher levels of cotton intoxication were observed (Fig. 3a). When cotton was sown on the day of the second application (0 days), all herbicide treatments promoted cotton intoxication, where 7 of the 14 herbicide treatments promoted plant death.

When the cotton was sown at 30 days, the lowest levels of intoxication were observed in treatments with 2,4-D and 2,4-D + glyphosate. At 60 days, in addition to the treatments already mentioned, the application of 2,4-D + saflufenacil, 2,4-D + glyphosate + saflufenacil, glyphosate + saflufenacil + [imazapic + imazapyr] and fluroxypyr also promoted intoxication of less than $10.0 \%$. In sowing performed at 90 and 120 days, only glyphosate + dicamba + saflufenacil treatment still provided above tolerable levels of intoxication.

All herbicide treatments provided lower plant height than the control plants when sowing was performed at 0 days (Fig. 3b). Only treatments with 2,4-D and 2,4-D + glyphosate no longer affected height when sowing was performed at 30 days. In sowing at 90 and 120 days, the only treatment that presented sufficient residual activity to affect the height of cotton plants was glyphosate + dicamba + saflufenacil.

Relative dry mass data of cotton plants are presented in Figure $3 c$. All herbicide treatments caused a reduction of more than $10.0 \%$ of the dry weight of cotton in relation to the control at sowing at zero days. For sowing at 30 days, only the 2,4-D and 2,4-D + glyphosate treatments no longer resulted in this reduction. The treatment that presented sufficient residual activity to affect the dry weight of cotton for longer period was glyphosate + dicamba + saflufenacil, providing significant reduction up to 120 days.

The SI estimated after two sequential applications of herbicide treatments evaluated in this work for cotton sowing are shown in Table 5. It is observed that with two sequential applications (A/B) the SI for most treatments increased. compared to the estimate for the single application (A) presented in Table 4. The lowest SIs calculated were 7 days for 2,4-D and 22 days for 2,4-D + glyphosate. The treatment containing dicamba in its composition presented SI over 120 days, which makes its use in the control of cotton stalks unfeasible if the objective is to cultivate cotton again in the following crop. Besides this treatment, the SI for cotton sowing after the application of herbicide treatments that had sulfentrazone, [imazapic + imazapyr], fluroxypyr or saflufenacil in combination was also close to or greater than 60 days.

Table 5. Estimated safety interval after two sequential applications of herbicide treatments (A/B) for cotton sowing based on phytointoxication, height and dry matter data. Experiment 2. Santo Antônio de Posse, SP, 2014.

\begin{tabular}{|c|c|c|c|}
\hline \multirow[t]{2}{*}{ Treatments (application A/B) } & \multicolumn{3}{|c|}{$\begin{array}{l}\text { Safety interval (SI) for the cotton sowing } \\
\text { (days after application) }{ }^{1}\end{array}$} \\
\hline & Phytointoxication & Height & Dry mass \\
\hline $2,4-D$ & 7 & 0 & 2 \\
\hline 2,4-D + glyphosate & 22 & 0 & 1 \\
\hline 2,4-D + saflufenacil & 54 & 39 & 31 \\
\hline 2,4-D + [imazapic + imazapyr] & 78 & 70 & 73 \\
\hline 2,4-D + glyphosate + saflufenacil & 43 & 47 & 60 \\
\hline 2,4-D + glyphosate + [imazapic + imazapyr] & 81 & 78 & 81 \\
\hline 2,4-D + glyphosate + saflufenacil + [imazapic + imazapyr $]$ & 79 & 75 & 80 \\
\hline 2,4-D + glyphosate + sulfentrazone & 79 & 78 & 83 \\
\hline 2,4-D + sulfentrazone & 87 & 69 & 65 \\
\hline Glyphosate + saflufenacil + fluroxypyr & 81 & 79 & 77 \\
\hline Glyphosate + [imazapic + imazapyr] + fluroxypyr & 77 & 70 & 78 \\
\hline Glyphosate + dicamba + saflufenacil & $>120$ & $>120$ & $>120$ \\
\hline Glyphosate + saflufenacil + [imazapic + imazapyr] & 46 & 43 & 52 \\
\hline Fluroxypyr & 46 & 32 & 51 \\
\hline Control without application & - & - & - \\
\hline
\end{tabular}


There was a significant difference in visual intoxication provided by the application of the 2,4-D and 2,4-D + glyphosate herbicide treatments, suggesting that when mixed (2,4-D + glyphosate) there is an increase in intoxication and prolongation of symptoms of injury on cotton plants. One possible explanation is the effect of some by-product that remained active in the soil from the interaction between the two herbicides, which may have caused an increase in the residual 2,4-D herbicide. Also, microbial degradation is the key process in the degradation of most soil herbicides, being valid for both glyphosate (ANDRIGHETTI et al., 2014) and 2,4-D (ARAÚJO; ORLANDA, 2014). When these herbicides are applied in combination there may be a possible overload of soil microbiota, which slows down herbicide degradation and provides greater persistence.

The presence of [imazapic + imazapyr], sulfentrazone or dicamba in the herbicide treatments provided enough residual activity to affect the cotton sown for a period longer than that determined by the host-free period. The same was observed when the herbicides fluroxypyr and saflufenacil were applied in combination (glyphosate + saflufenacil + fluroxypyr). The cotton host-free period may vary, depending on the producing state, but it is usually adopted at least 60 days between the beginning and the end of this period. In the state of Mato Grosso, for example, it starts between October $1^{\text {st }}$ and October $15^{\text {th }}$, and lasts for 60 days, ending on November $30^{\text {th }}$ to December $14^{\text {th }}$, depending on the region (AMPA, 2020).

Imazapic and imazapyr belong to the imidazolinone chemical group and have long soil persistence (RODRIGUES; ALMEIDA, 2018). MONQUERO et al. (2010) observed that imazapyr affected susceptible plants for periods of 150 to 210 days. The 2,4-D + [imazapic + imazapyr] treatment, for example, presented SI between 78 and 90 days for cotton sowing, which corroborates the results obtained by MATTE et al. (2018), who did not observe any negative effects on cotton sown 112 DAA of [imazapic + imazapyr], which is longer than the SI obtained in the present study.

Sulfentrazone also has persistence in the soil for long periods, which increases the risk of affecting successive crops, as observed in this work. Sulfentrazone half-life in the soil is estimated to be between 110 and 280 days, varying according to local edaphoclimatic conditions (VIVIAN et al., 2006). The SI for cotton sowing after application of 2,4-D + sulfentrazone treatment, for example, was 87 to 89 days. However, MATTE et al. (2019) observed that sulfentrazone had enough residual activity to cause intoxication of sown cotton 112 DAA.

Although dicamba is an auxinic herbicide like 2,4-D, it can be seen in this work that its residual effect on the soil was much higher. Dicamba has higher water solubility, lower Kow value (octanol-water partition coefficient) and lower soil sorption value $(\mathrm{Kd})$ or organic matter $(\mathrm{Koc})$, which may favor higher herbicide availability in the soil solution and, consequently, present higher residual activity in relation to other auxinic herbicides (OSIPE et al., 2014; RODRIGUES; ALMEIDA, 2018; VILLAVERDE et al., 2008). According to BUNCH et al. (2020), its half-life may vary from 30 to 60 days. Its characteristics may have contributed to the appearance of injuries up to 100 days after single application of glyphosate + dicamba + saflufenacil mixture and more than 120 days after sequential application.

The results obtained in this work may have overestimated the withdrawal period for some herbicides. This is because the experiments were conducted in a greenhouse, simulating a possible condition that occurs in the field. Also, between the treatment application and cotton sowing, the pots received no irrigation, which may result in slower herbicide degradation in the soil. Thus, the results provide indicative of the residual activity of these herbicides that may help in the adoption of strategies of management of cotton stalks in compliance with the host-free period, aiming the cultivation of cotton in the next crop.

The way this work was conducted, it concluded that the single application and sequential application of the treatments that present in their composition dicamba, sulfentrazone and [imazapic + imazapyr], in addition to the association of glyphosate + saflufenacil + fluroxypyr, can provide residual activity. sufficient to affect the cotton sown after the host-free period, with SI greater than 60 days. The 2,4-D and 2,4-D + glyphosate treatments were the safest, with the shortest SIs even in sequential applications, not promoting enough residual activity to affect cotton sowing after the host-free period. 


\section{AUTHORS' CONTRIBUTIONS}

Conceptualization: Francischini, A.C.; Constantin, J.; Oliveira Junior, R.S. Data curation: Francischini, A.C.; Matte, W.D. Formal analysis: Francischini, A.C.; Constantin, J.; Oliveira Junior, R.S.; Matte, W.D. Funding acquisition: Constantin, J.; Oliveira Junior, R.S. Investigation: Francischini, A.C.; Constantin, J.; Oliveira Junior, R.S.; Methodology: Francischini, A.C.; Constantin, J.; Oliveira Junior, R.S. Project administration: Francischini, A.C.; Constantin, J.; Oliveira Junior, R.S. Resources: Francischini, A.C.; Matte, W.D. Supervision: Constantin, J.; Oliveira Junior, R.S. Validation: Mendes, R.R.; Machado, F.G. Visualization: Francischini, A.C.; Constantin, J.; Oliveira Junior, R.S.; Matte, W.D.; Mendes, R.R.; Machado, F.G. Writing - original draft: Francischini, A.C. Writing review \& editing: Matte, W.D.; Mendes, R.R.; Machado, F.G.

\section{AVAILABILITY OF DATA AND MATERIAL}

The datasets generated and/or analyzed during the current study are available from the corresponding author on reasonable request.

\section{FUNDING}

Coordenação de Aperfeiçoamento de Pessoal de Nível Superior (CAPES)

https://doi.org/10.13039/501100002322

Finance Code 001

\section{CONFLICTS OF INTEREST}

The authors certify that they have no commercial or associative interest that represents a conflict of interest in connection with the manuscript.

\section{ETHICAL APPROVAL}

Not applicable.

\section{ACKNOWLEDGEMENTS}

To all members of the Núcleo de Estudos Avançados em Ciência das Plantas Daninhas at the Universidade Estadual de Maringá (NAPD/UEM), Brazil.

\section{REFERENCES}

ALMEIDA, R.P.; SILVA, C.A.D.; RAMALHO, F.S. Manejo integrado de pragas do algodoeiro no Brasil. In: BELTRÃO N.E. de M., AZEVEDO D.M.P. de (ed.). O agronegócio do algodão no Brasil. Brasília: Embrapa Informação Tecnológica, 2008.

AMPA - Associação Matogrossense dos Produtores de Algodão. Vazio sanitário do algodão começa em outubro. Available from: http:// www.https://ampa.com.br/vazio-sanitario-do-algodao-comeca-em-outubro/. Access on: 2 Feb. 2020.

ANDRADE JUNIOR, E.R. de; CAVENAGHI, A.L.; GUIMARÃES, S.C. Destruição química de soqueira em variedades de algodoeiro resistentes ao glifosato - Safra 2016 (Circular Técnica IMAmt n.29). Primavera do Leste: IMAmt, 2017. Available from: https://upherb. com.br/ebook/circular_tecnica_IMA_29.pdf. Access on: 3 Feb. 2020.

ANDRIGHETTI, M.S.; NACHTIGALL, G.R.; QUEIROZ, S.C.N. de; FERRACINI, V.L.; AYUB, M.A.Z. Biodegradação de glifosato pela microbiota de solos cultivados com macieira. Revista Brasileira de Ciência do Solo, Viçosa, v.38, n.5, p.1643-1653, 2014. https://doi. org/10.1590/S0100-06832014000500029

ARAÚJO, L.C.A. de; ORLANDA, J.F.F. Biodegradação do herbicida 2,4-D utilizando bactérias selecionadas do solo do cerrado maranhense. Pesticides: Revista de Ecotoxicologia e Meio Ambiente, Curitiba, v.21, p.21-32, 2014. Available from: https://revistas.ufpr.br/pesticidas/ article/view/39011/23773. Access on:5 Feb. 2020.

BUNCH, T.R.; GERVAIS, J.A.; BUHL, K.; STONE, D. Dicamba technical fact sheet. Corvallis: NPIC, 2012. Available from: http://npic. orst.edu/factsheets/archive/dicamba_tech.html. Access on: 4 Jan. 2020.

FERREIRA, A.C. de B.; BORIN, A.L.D.C.; BRITO, G.G. de; SILVA FILHO, J.L. da; BOGIANI, J.C. Épocas de semeadura, cultivares e densidades de plantas para algodão adensado em segunda safra. Pesquisa Agropecuária Tropical, Goiânia, v.45, n.4, p.397-405, 2015. https://doi.org/10.1590/1983-40632015v4536869 
FRANCISCHINI, A.C.; CONSTANTIN, J.; MATTE, W.D.; OLIVEIRA JUNIOR, R.S.; RIOS, F.A.; MACHADO, F.G. Association of mechanical and chemical methods for cotton stalk destruction. Planta Daninha, Viçosa, v.37, e019207486, 2019. https://doi.org/10.1590/ s0100-83582019370100141

GHENO, E.A.; OLIVEIRA JUNIOR, R.S. de; CONSTANTIN, J.; TAKANO, H.K.; GEMELLI, A. Residual activity of herbicides applied to cotton on crops cultivated in succession. Revista Caatinga, Mossoró, v.29, n.1, p.143-152, 2016. https://doi.org/10.1590/1983-21252016v29n117rc

GRIGOLLI, J.F.J.; CROSARIOL NETTO, J.; IZEPPI, T.S.; SOUZA, L.A. de; FRAGA, D.F.; BUSOLI, A.C. Infestação de Anthonomus grandis (Coleoptera: Curculionidae) em rebrota de algodoeiro. Pesquisa Agropecuária Tropical, Goiânia, v.45, n.2, p.200-208, 2015. https://doi. org/10.1590/1983-40632015v4532296

MATTE, W.D.; CAVALIERI, S.D.; PEREIRA, C.S.; IKEDA, F.S; POLTRONIERI, F. Residual activity of [imazapic+imazapyr] applied to soybean resistant imidazolinones on cotton in succession. Planta Daninha, Viçosa, v.36, e018181240, 2018. https://doi.org/10.1590/ s0100-83582018360100148

MATTE, W.D.; CAVALIERI, S.D.; PEREIRA, C.A; IKEDA, F.S; SHENG, L.Y. Residual activity of sulfentrazone applied to soybean on cotton crop in succession. Planta Daninha, Viçosa, v.37, e019187015, 2019. https://doi.org/10.1590/s0100-83582019370100043

MONQUERO, P.A.; SILVA, P.V.; HIRATA, A.C.S.; TABLAS, D.C.; ORZARI, I. Lixiviação e persistência dos herbicidas sulfentrazone e imazapic. Planta Daninha, Viçosa, v.28, n.1, p.185-195, 2010. https://doi.org/10.1590/S0100-83582010000100022

OLIVEIRA, M.F. de; BRIGHENTI, A.M. Comportamento de herbicidas no ambiente. In: OLIVEIRA JUNIOR, R.S. de; CONSTANTIN, J.; INOUE, M.H. (ed.). Biologia e manejo de plantas daninhas. Curitiba: Omnipax, 2011. chap.11, p.263-304.

OSIPE, J.B.; OLIVEIRA JUNIOR, R.S. de; CONSTANTIN, J.; BIFFE, D.F.; RIOS, F.A.; FRANCHINI, L.H.M.; GHENO, E.A.; RAIMONDI, M.A. Seletividade de aplicações combinadas de herbicidas em pré e pós-emergência para a soja tolerante ao glyphosate. Bioscience Journal, Uberlândia, v.30, n.3, p.623-631, 2014. Available from: https://docs.bvsalud.org/biblioref/2018/10/947177/seletividade-de-aplicacoescombinadas-de-herbicidas-em-pre-e-po_PDsIar8.pdf. Access on: 7 Feb. 2020.

RIBEIRO, E.B.; CASTELLANI, M.A.; SILVA, C.A.D. da; MELO, T.L.; SILVA, G. dos S., VALE, W.S. do; SANTOS, A.S. Métodos de destruição de restos de cultura do algodoeiro e sobrevivência do bicudo. Pesquisa Agropecuária Brasileira, Brasília, v.50, n.11, p.993-998, 2015. https://doi.org/10.1590/S0100-204X2015001100001

RODRIGUES, B.N.; ALMEIDA, F.S. Guia de herbicidas. Londrina: Plantarum, 2018. 764p.

VILLAVERDE, J.; KAH, M.; BROWN, C.D. Adsorption and degradation of four acidic herbicides in soils from Southern Spain. Pest Management Science, London, v.64, n.7, p.703-710, 2008. https://core.ac.uk/download/pdf/36136652.pdf. Access on: 10 Feb. 2020.

VIVIAN, R.; REIS, M.R.; JAKELAITIS, A.; SILVA, A.F.; GUIMARÃES, A.A.; SANTOS, J.B.; SILVA, A.A. Persistência de sulfentrazone em argissolo vermelho-amarelo cultivado com cana-de-açúcar. Planta Daninha, Viçosa, v.24, n.4, p.741-750, 2006. https://doi.org/10.1590/ S0100-83582006000400015 